\title{
Web Enabled High Fidelity Drilling Computer Model with User- Friendly Interface for Education, Research and Innovation
}

\author{
Robert Ewald ${ }^{1}$, Jan Einar Gravdal ${ }^{1}$, Dan Sui ${ }^{2}$, Roman Shor ${ }^{3}$ \\ ${ }^{1}$ IRIS - International Research Institute of Stavanger, Norway, ${ }^{2}$ University of Stavanger, Dept. of Petroleum Technology, \\ ${ }^{3}$ University of Calgary, Dept. of Chemical and Petroleum Engineering, Canada
}

\begin{abstract}
Next generation intelligent software for drilling control systems together with automated monitoring and analysis systems is expected to save costs for the drilling industry. However, the transition from monitoring a process, which today is controlled manually, to automating the process requires a step-change in education of personnel as well as in infrastructure for development and testing new technology. The lack of high quality field data from drilling and well operations is a major problem in research and innovation projects within the oil \& gas and geothermal drilling sector, as well as in education within these areas. Since 2015, IRIS and the University of Stavanger have developed a web enabled high fidelity drilling simulator as part of the OpenLab Drilling project ${ }^{1}$.

This paper describes the objectives of the project, the technical solutions of the web enabled drilling simulator, and the results obtained during the first year after deployment to the users.
\end{abstract}

Keywords: $\quad$ Process modeling, Well construction, Process control, Simulator, Web Interface

\section{Introduction}

During a drilling operation large amount of data is collected. However, the amount of information that can be retrieved from the data is often sparse, and the efforts to use the data for analysis and process control purposes is very challenging (Arnaout et al 2013; Ashok, et al 2016; Maidla et al 2018; Cayeux et al, 2013a). The lack of high-quality data and information about the data (metadata) also hinders technology development and demonstration of innovative solutions in realistic environments. However, technology can be tested and demonstrated in a simulated environment up to a certain technology readiness level. IRIS, together with Statoil and Sekal ${ }^{2}$ has experience with the DrillTronics ${ }^{\mathrm{TM}}$ system where advanced drilling control software has been tested extensively through many years in a

\footnotetext{
${ }^{1}$ OpenLab Drilling (formerly known as Virtual Arena) is an infrastructure project funded by the Research Council of Norway: www.openlabdrilling.com
}

simulated environment (Cayeux et al 2012). Worldwide, there are several drilling simulators available that include both transient hydraulics- and drill string mechanic computations. In Norway, research and development at Rogaland Research (later known as IRIS) in the 1980's and 90's paved way for the well flow model RF-Kick (Petersen et al, 1998), later commercialized in DrillBench (which later became part of a Schlumberger Software suite). The RF-Kick was verified by data collected from Ullrigg in Stavanger and validated through various test campaigns both at Ullrigg and other wells (Rommetveit et al 1991). The model was used for planning and special studies for drilling operations. From 2001, Rogaland Research initiated a large program to improve their well flow model and prepare for real-time (online) simulations. A new numerical solver was developed (Lorentzen et al 2002) and the well flow model was integrated with other models such as a torque $\&$ drag model (Yi et al 2004) and transient cuttings transport model (Cayeux et al 2013b), and recently a transient torque \& drag model (Cayeux et al 2018). This simulation model is commonly known as WeMod. Another simulation model was developed during the 2000 s by SINTEF (Petersen et al 2008). This model, together with a torque $\&$ drag model is now used in software for training and real-time monitoring (Ødegård et al 2013) now commercialized by eDrilling ${ }^{3}$. Another renown well flow model is the simplified well flow model developed at the Norwegian University of Science and Technology and Statoil by Kaasa et al. (2012). A thorough review of these and other models are given by Aarsnes et al (2014).

Common for software based on simulation models developed at the research communities is that the user interface is designed for scientists and engineers skilled to set up the cases (configure the model), run the software and analyze the results. This has the benefit that the complexity of the models can be exploited to their full potential. However, this is at the expense of

\footnotetext{
2 Sekal is a Norwegian company owned by Statoil, Saudi Aramco and IRIS: www.sekal.com

${ }^{3}$ eDrilling is a Norwegian Company owned by Teresoft.
} 
user friendliness, and makes it difficult for non-experts to utilize such models.

\section{Project objective}

The overall objective of the OpenLab Drilling project is to stimulate education, research and innovation and to increase the acceptance and uptake of new technologies within automated drilling. One of the secondary objectives is to create a user-friendly interface to high fidelity models so that realistic (but artificial) data can be available for students, lecturers, and analysts (researchers and engineers). This is the motivation behind the development of the web enabled drilling simulator.

\section{OpenLab simulator}

The simulator uses IRIS WeMod as the core model, with its high fidelity transient well flow and drill string vibrational model. Because of the advantages over desktop applications, a web-based solution has been preferred. Among the benefits that have been considered are:

- Cost effective development

- Accessible anywhere

- Easily customizable

- Accessible from a range of devices

- Improved interoperability

- Easier installation and maintenance

- Adaptable to increased workload

- Increased Security when deployed on dedicated servers

- Promotes e-learning and thereby more efficient and environment friendly collaboration which reduces the need for travel.

The motivation by developing the web-based architecture is that a software available through a web application programming interface (API) has the potential to become an easily accessible software for students, lecturers and analysts. To reach out to as many users as possible the OpenLab Drilling project has focused on user friendliness for students, lecturers and analysists, and for those who are not experienced with drilling simulation software. The graphical user interface (GUI) has therefore been developed in close collaboration with drilling engineers as well as unexperienced students and lecturers of drilling courses and experienced researchers.

\section{User groups}

To guide the design of the user interaction it was early decided which user groups to support and how these should be prioritized. This has guided the development team to make decisions when conflicting solutions have been proposed. Each user group has specific needs and skills, which have been assessed through interviews and user tests.
Students

- are not familiar with the industrial way of presenting drilling data

- $\quad$ are familiar with web interaction paradigms

- need to learn how inputs to the drilling system affect drilling operations

Lecturers

- desire simple to use but physically accurate scenario-based simulations

- prefer that students spend time understanding the scenario rather than the simulation system

- benefit from systems which allow easy collaboration or knowledge sharing

- demonstrate drilling simulations in different scenarios for classroom teaching

- design projects, tasks \& assignments in an easy way

- need for the teaching with digital technologies

Analysts/Engineers

- collaborate to a larger extent with other users.

- access various configurations and simulations.

Although these user groups and their individual users will have different needs and expectations to a drilling simulator, there are some common requirements that needed to be met. These are mainly related to user friendliness in configuration and simulation, to uptime and robustness of the software, and to access and storage of configurations and results.

\section{User friendliness}

The complex configuration needed to run high-fidelity simulators is a barrier to entry. Such models require a huge amount of configuration parameters to be set. A configuration in the Web Enabled Drilling Simulator is a description of the different elements and components that affect the circulation system and drillstring mechanics. In OpenLab Drilling this challenge has been addressed with a few complementing design decisions: Adequate defaults, deliberate choice of interaction components, and extensive input validation. Selection of default configurations have a big impact to lower the barrier of entry. A simple choice of preconfigured wells which cover different use cases is therefore presented for the user at entry. Each of these is ready to be simulated interactively. The user is guided through the configuration of casing architecture, trajectory, drill string design, drilling fluid parameters, geo-pressures and geothermal gradient. All of which comes with default configuration parameters. This allows learning the application in a step-by-step fashion while remaining sufficient flexibility for experienced users to input complex scenarios. 


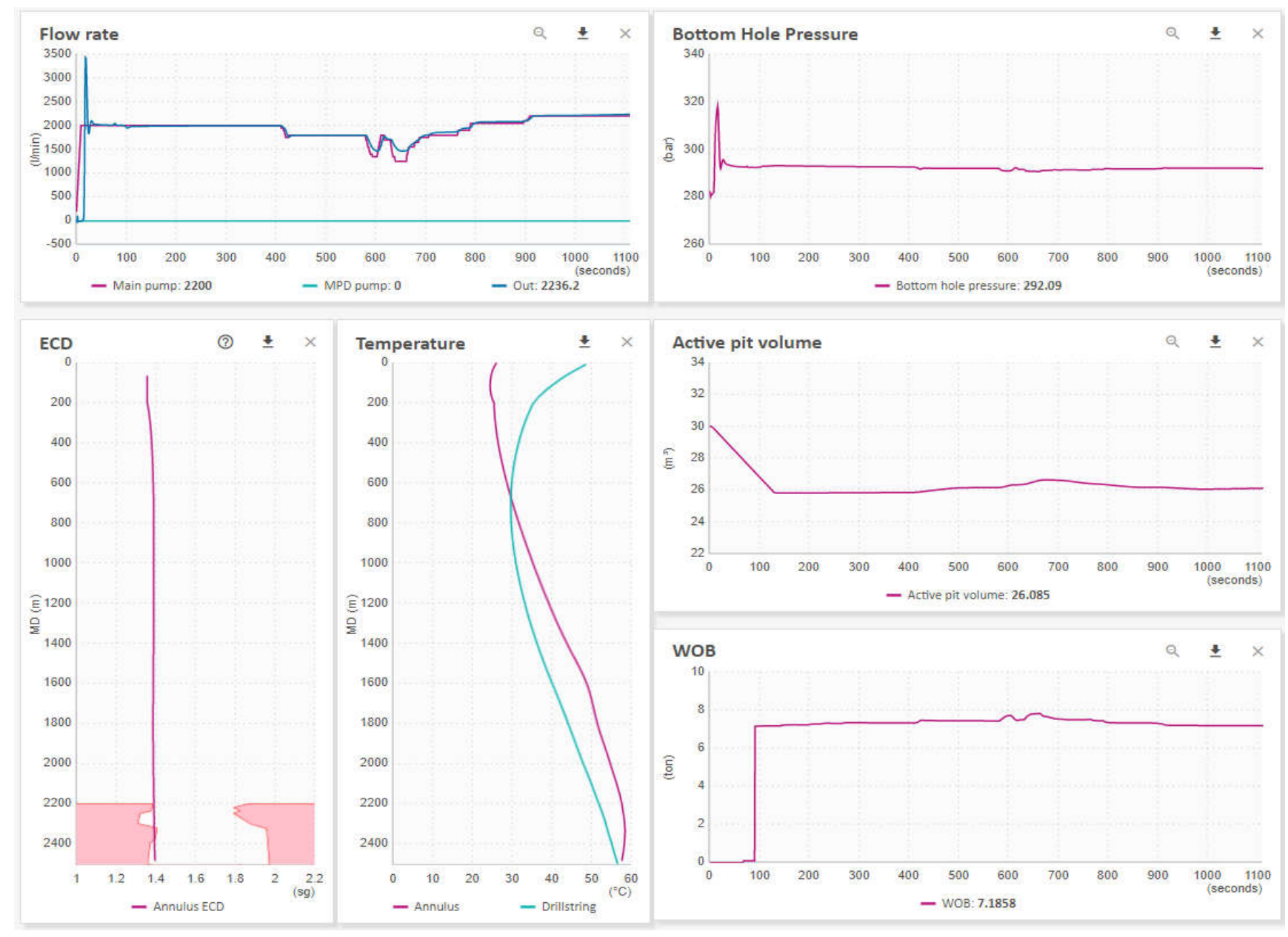

Figure 1: Simulation results are available in graphs from which data can be downloaded.

\section{Input Validation}

Although adequate defaults are provided, the user may adjust many interacting configuration options and setpoints. Without any validation of configurations and simulation setpoints, inconsistent options lead to crashing the simulator. This is unacceptable since the user is not informed about the inner workings of the simulator. To remedy this, a validation layer is included which catches conflicting configuration parameters which might crash the simulator. Nevertheless, the default configurations can be changed completely based on the user's own specifications. A change in a configuration may lead to validation errors that comes together with a message describing precisely which variables that need to be changed to regain validity. A configuration is not approved for simulation until all validation errors are removed by the user.

The validation and the quick feedback it enables, teaches the user how drilling data is related. With a valid configuration it is guaranteed that a simulation can be initialized and started.

\section{Simulations}

OpenLab Drilling is flexible for a user to simulate a wide range of scenarios such as well control, hole cleaning problems, buckling, and drill string vibrations to mention a few. To run a new simulation, a few initializations parameters need to be entered. The initial depth of the drill bit defines a starting point for the simulation. When running a simulation several setpoints (manipulated variables) can be edited at any time to simulate a drilling process. These are related to (1) fluid flow, (2) drill string movements, and (3) choke openings.

The setpoints are constrained by upper and lower bounds to prevent the user from entering unphysical values or values that may crash the simulator. The validation of configuration parameters and setpoints guarantees that a simulation will start and run. However, as for real drilling operations the user (or driller) can end up in a situation where only careful adjustment of the drilling parameters will prevent the drill string to become stuck in the borehole, or severe gas kicks to destabilize the well. A simulation can be run into a state where the models can no longer produce a reliable result and eventually crash. This can e.g. be the 
case when large amounts of cuttings accumulate in the well because of insufficient hole cleaning, and eventually creates a completely blocking of the annulus. It should be emphasized that each simulation is an individual process and a crashed simulation will of course not affect other simulations running concurrently.

In simulation mode, all relevant time- and depthbased plots are available in the web browser on the user's demand and can suit the scenario at hand. During simulation, the user controls all the main drilling parameters at 1 Hertz time resolution. The simulator can also run in fast-forward which typically implies 10 times real time dependent on the client computer's performance and the network latency. The simulation process can be paused, perform a single step, or completed and shut down. A simulation can also run in sequential mode in which a predefined selection of setpoints is entered in a table. The user can then specify changes in any setpoint at a given time and construct a sequence. When executing a sequence, the user cannot interact with the simulator and change any of the setpoints. When the simulator runs in sequence mode, the simulation speed is typically around 15 times real time. The benefit by the sequential mode is that the user can run through the same scenario on different configurations and explore how a certain procedure will evolve for different wells, drilling fluids, etc.

Simulation results can be downloaded at any time during or after a simulation from the simulation GUI. For simplicity all results are downloaded to CSV format for easy processing in e.g. Excel. As for the configurations, the simulations can be shared. Unlike other desktop applications, a web enabled simulator allows other people from anywhere in the world to monitor and interfere with the simulation. By sending the URL a user can invite others to monitor an ongoing simulation or explore the results on completed simulations. The receiver of a URL can also take control and run a simulation on behalf of the owner of the simulation.

In addition to the browser interface, a WebAPI is available, making the simulator accessible to Matlab ${ }^{\mathrm{TM}}$ and Python, among others. These interfaces require special knowledge about the particular programming language and a description of these interfaces is not part of this paper.

\section{Architecture and concept of operation}

The system architecture of the OpenLab Drilling simulation system needs to account for performance and security. For this reason, the following system components are used:

- Webserver which serves the WebAPI

- Watchdog service per simulation server
- Relational database

- Clients: Webbrowser, Matlab client or Python client. Other clients may be developed by external users of the WebAPI.

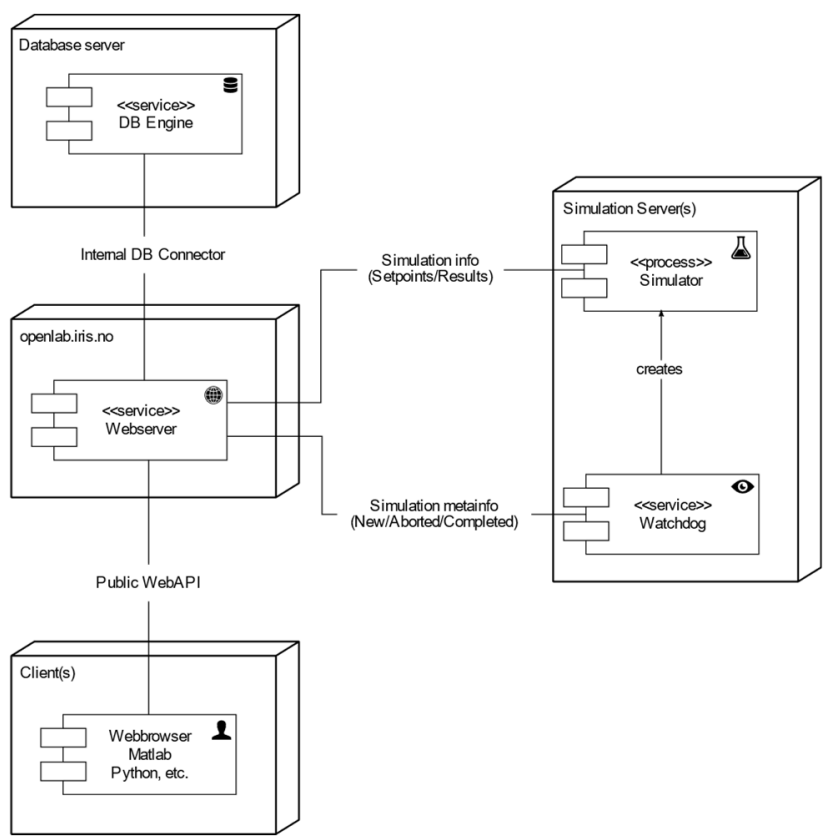

Figure 2: System communication architecture

To support a continuous development process, a Build server and a Deployment server has been established. By this approach a documented and consistent deployment is possible which makes it easy to release small improvements relatively often.

Immediate feedback for users is an important enabler of learning. Consequently, keeping the latency of the system low is important. The load on the system depends on the number of simulations which run concurrently and the number of users monitoring the results. The system works as follows: Watchdog processes monitor the WebAPI for new simulation requests. One watchdog then claims responsibility for this request and starts a simulation process. If the cores of the simulation machine are busy, the watchdog will respond slower to a simulation request and other watchdog processes with less utilized cores will pick up the simulation request. By this approach an automated load balancing is achieved without explicitly assigning work to different machines. In addition, a new simulation machine can be connected to the WebAPI and immediately assist when starting new simulations.

When a simulation process has been created and is initialized, it picks step requests from a queue and processes them as quickly as it can. The request contains the boundary conditions called setpoints. When the timestep is simulated the results are sent to the WebAPI which is responsible to persist them in the database.

The WebAPI is responsible for connecting user facing clients and simulation clients. It also persists 
configurations, setpoints and results to make simulations reproducible. The WebAPI enforces authorization as well.

Since the system is exposed to different users with different trust levels, the architecture addresses security concerns. Malicious data may be used to subvert parts of the system and threaten assets that need to be protected. A main tool to mitigate threats is isolation of components using operating system tools such as process isolation and firewalls.

The most complex component of the system is the simulation which may crash when input data are invalid. This makes various exploits possible and a malicious user may subvert the simulation process for other purposes.

This risk is mitigated by validating data, and also by isolating each simulation process and allowing the communication of results only to the WebAPI.

\section{The WeMod simulation model}

The IRIS WeMod simulation model can simulate a wide range of downhole effects with high fidelity. The simulator consists of a set of fully integrated numerical models:

- A transient multi-phase flow model that solves mass and momentum balance to estimate pressure distribution inside the wellbore. (Lorentzen et al 2002 and 2014). The flow model has been improved, tested and validated over nearly 20 years. Dynamic effects such as pump accelerations, drill-string movements (surge-swab), gelling, and the presence of several drilling or formation fluids in the well are simulated. Flow of hydrocarbon to and from the formation is calculated by an integrated near-well reservoir model.

- A transient cuttings transport model that estimates the distribution of the cuttings inside the annulus and determines whether the drilled solids are in suspension in the drilling fluid or accumulate in cuttings bed (Cayeux et al 2013b). Transport of cuttings by bed erosion is also simulated.

- A torque and drag model that computes the tension and torque distribution along the drillstring (Yi et al 2004). In the current version a soft string model is used, however during 2018 a new transient torque and drag model will be available.

- A heat transfer model, which computes the temperature evolution inside the wellbore and in the formation near the well (Corre et al, 1984). Forced convection, heat conduction, and convective heat transfer are accounted for in the simulations.
The above-mentioned models are integrated in such a way that the hydraulic model uses the temperature profile generated by the heat transfer model to estimate pressures, densities and velocities in a numerical grid. The results are in turn used by the torque and drag model for buoyancy calculations and by the cuttings transport model for estimation of the transport capabilities. For density and rheology estimations, the hydraulic model uses the proportion of cuttings in suspension generated by the cuttings transport model.

\section{Future extensions}

OpenLab Drilling is currently providing simulated drilling data. The applications are hydrocarbon and geothermal drilling. However, for most users of OpenLab Drilling the access to real drilling data will be of high value. For analysts and engineers, the ability to test a prototype software at an early stage on real data will be of high importance and potentially affect the robustness and usability of the final product greatly. For students, access to real data will give valuable insight to the actual precision of the drilling data and provide a much more realistic view of the challenges the industry is facing when evolving towards drilling automation. A natural extension of OpenLab Drilling is therefore to offer an interface to providers and users of real drilling data.

It is also the intention to extend OpenLab to other disciplines than drilling, such as reservoir simulation and production data. The architecture developed in the OpenLab project is suitable for such use and can facilitate more knowledge transfer between the disciplines.

\section{Project results}

The drilling industry's increased focus on cost reduction has paved the way for automation in terms of process control rather than machine control. Process control in drilling requires technology developed to control a complex, non-linear and highly transient process. Fast and accurate simulation models that can predict downhole conditions accurately are therefore becoming more important. Until now, the user interface of simulators like WeMod and other high-fidelity simulators has been overwhelming for students and other novice users and erects a high barrier to onboard new users. With OpenLab Drilling an interface is made available for all who has a need for realistic but artificial drilling data provided by a high-fidelity simulator. The user interface allows users with very limited experience to drilling to take it into use. OpenLab Drilling is now being used as part of regular drilling courses at the University of Stavanger and the University of Calgary. In addition, Master and $\mathrm{PhD}$ students at other universities in Norway and abroad are using the simulator. Two of the major oil \& gas operators in 
Norway are using the software. During the first months when the software has been available in 2018 nearly 100 new users have taken the software into use.

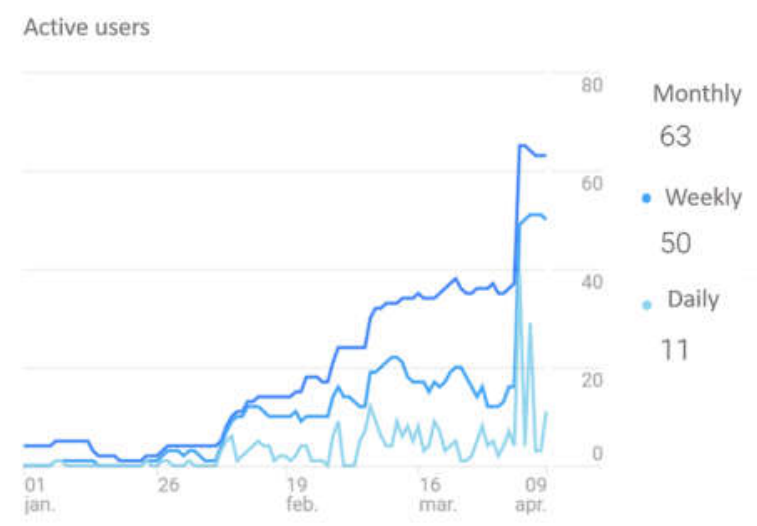

Figure 3: Registered user of OpenLab from January $1^{\text {st }}$ to April $9^{\text {th }} 2018$. Ref: Google Analytics.

\section{Conclusions}

In the OpenLab Drilling project a drilling simulator on the web has been developed for students, educators and analysts with a low barrier of entry. The web enablement has made it possible to deploy the software to many users during the development phase to obtain valuable feedback at an early stage. Thanks to this, the user interface and prioritized functionally is continuously developing to meet the users' needs. The system is now deployed at universities in Norway and abroad, and to major oil \& gas operators. Close collaboration with the user groups has been a key to succeed in developing a user interface that fits the need from students, researchers and lecturers.

\section{Acknowledgements}

The authors acknowledge the Research Council of Norway for financing OpenLab Drilling through the National Financing Initiative for Research Infrastructure (INFRASTRUKTUR). The authors are also very grateful to Miles AS in Stavanger, and CloudService AS for providing IT competence to the project.

\section{References}

Aarsnes, U.J.F, NTNU, Di Meglio, F., Mines ParisTech, Aamo, O.M., NTNU, Kaasa, G-O.Statoil. Fit-for-purpose Modeling for Automation of Underbalanced Drilling Operations. Paper SPE/IADC 168955. SPE/IADC Managed Pressure Drilling \& Underbalanced Operations Conference \& Exhibition, 8-9 April, Madrid, Spain, 2014.

Arnaout, A., Zoellner, P., and Thonhauser, G., TDE Tonhauser Data Engineering $\mathrm{GmbH}$, Johnstone, N. "Intelligent Data Quality control of Real-time Rig Data." SPE 167437. SPE Middle East Intelligent Energy
Conference and Exhibition, 28-30 October, Manama, Bahrain, 2013.

Ashok, P., Ambrus, A., Ramos, and D., Lutteringer, J., Sentinel Real time LLC, and Behounek, M., Yang, Y. L., Thetford, T., and Weaver, T., Apache Corp. "A Step by Step Approach to Improving Data Quality in Drilling Operations: Field Trials in North America." SPE 181076., SPE Intelligent Energy International Conference and Exhibition, 6-8 September, Aberdeen, Scotland, UK, 2016.

Cayeux, E., Daireaux, B., Dvergsnes, E.W., and Leulseged, A., IRIS, Bruun, B., Statoil, and Herbert, M., ConocoPhillips Norway. "Advanced Drilling Simulation Environment for Testing New Drilling Automation Techniques". SPE 150941. IADC/SPE Drilling Conference and Exhibition, 6-8 March, San Diego, California, USA, 2012.

Cayeux, E., Daireaux, B., Dvergsnes, E. W., IRIS. Florence, F., National Oilwell Varco. "Toward Drilling Automation: On the Necessity of Using Sensors That Relate to Physical Models". Paper SPE 163440. SPE/IADC Drilling Conference, 5-7 March, Amsterdam, The Netherlands, 2013.

Cayeux, E., IRIS, Mesagan, T., Tanripada, S., Zidan, M., Statoil, Fjelde, K. K., University of Stavanger. "Real-Time Evaluation of Hole Cleaning Conditions Using a Transient Cuttings Transport Model". Paper SPE 163492. SPE/IADC Drilling Conference, 5-7 March, Amsterdam, The Netherlands, 2013.

Cayeux, E. IRIS. “On the Importance of Boundary Conditions for Real-Time Transient Drill-String Mechanical Estimations". Paper SPE 189642. IADC/SPE Drilling Conference and Exhibition, 6-8 March, Fort Worth, Texas, USA, 2018.

Corre, B., Eymard, R. and Gounet, A. "Numerical computation of Temperature Distribution in a wellbore while drilling". Paper SPE 132080 presented at the SPE Annual Technical Conference and exhibition, Houston, 1619 September, 1984.

Kaasa, G-O, Statoil, Stamnes, Ø.N., Aamo, O.M. and Imsland, L.S., Norwegian University of Science and Technology. Paper SPE 143097. SPE Drilling \& Completion Journal. Vol. 27, Issue 01. March 2012.

Lorentzen, R.J., RF-Rogaland Research. "Higher Order Numerical Methods and Use of Estimation Techniques to Improve Modeling of Two-Phase Flow in Pipelines and Wells". Doctor thesis, University of Bergen, April 2002.

Lorentzen, R.J., Stordal, A. and Nævdal, G., SPE, IRIS, and Karlsen, H.A. and Skaug, H.J., University of Bergen, Department of Mathematics. Estimation of Production Rates with Transient Well-Flow Modeling and the Auxiliary Particle Filter. Paper SPE 165582, SPE Journal, pages 172-180, February 2014.

Maidla, E., Maidla, W., Rigg, J., Crumrine, M., WolfZoellner, P., ProNova - TDE Petroleum Data Solutions, Inc. "Drilling Analysis Using Big Data has been Misused and Abused". Paper SPE 189583. IADC/SPE Drilling Conference and Exhibition, 6-8 March, Fort Worth, Texas, USA, 2018.

Petersen, J. and Rommetveit, R., RF-Rogaland Research, Tarr, B., Mobil Technology Company. "Kick with Lost Circulation Simulator, a Tool for Design of Complex Well 
Control Situations”. Paper SPE 49956. SPE Asis Pacific Oil \& Gas Conference and Exhibition, Perth, Australia, 12-14 October 1998.

Petersen, J., Rommetveit, R., Bjorkevoll, K.S., Froyen, J., SINTEF Petroleum Research. "A General Dynamic Model for Single and Multi-Phase Flow Operations during Drilling, Completion, Well Control and Intervention". Paper SPE 114688. IADC/SPE Asia Pacific Drilling Technology Conference and Exhibition, 25-27 August, Jakarta, Indonesia, 2008.

Rommetveit, R., Vefring, E.H. RF-Rogaland Research. «Comparison of Results from and Advanced Gas Kick Simulator with Surface and Downhole Data From Full Scale Gas Kick Experiments in an Inclined Well". Paper SPE 22558. SPE Annual Technical Conference and Exhibition, Dalla, TX, USA, 6-9 October 1991.

Yi, Y., Lund, B., Aas, B., He, X., Rommetveit, R., Lazaro, D., Stokka, S., RF-Rogaldn Research. Bottazzi, F., ENI. "An Advanced Coiled Tubing Simulator for Calculations of Mechanical and Flow Effects; Model Advancements and Full-Scale Verification Experiments." Paper SPE 89455, SPE/ICoTA Coiled Tubing Conference and Exhibition, Houston, TX, USA, 23-24 March 2004.

Ødegard, S.I, eDrilling, Risvik, B.T., Statoil, Bjorkevoll, K.S., SINTEF, Mehus, Ø., Oiltec Solutions, Rommetveit, R and Svendsen, M., eDrilling. "Advanced Dynamic Training Simulator for Drilling as well As Related Experience from Training of Drilling Teams with Focus on Realistic Downhole Feedback". Paper SPE 163510. SPE/IADC Drilling Conference, 5-7 March, Amsterdam, The Netherlands, 2013. 